Z. klin. Chem. u. klin. Biochem.

9. Jg., S. $520-526$, November 1971

\title{
Bestimmung von 11-Hydroxy- und 11-Desoxycorticoiden aus der gleichen Plasmaprobe als Basis eines Metopironkurztestes
}

\author{
Von H. K. KLEY und H. L. KRÜSKEMPER ${ }^{1}$ ) \\ Medizinische Hocbscbule Hannover \\ ,
}

(Eingegangen am 14. Juni 1971)

\begin{abstract}
Eine einfache und schnell durchführbare Methode zur Bestimmung der Plasmakonzentrationen von 11-Hydroxycorticoiden, 11-Desoxycorticoiden und Gesamtcorticoiden nach Applikation von Metopiron wird beschrieben. Den Probanden werden in einem Kurztest $4 \mathrm{~g}$ Metopiron verteilt über 21/2 Stunden gegeben. Die 11-Desoxycorticoide und die 11-Hydroxycorticoide werden aus $200 \mu 1 \mathrm{Plasma}$ durch eine zweistufige Extraktion mit Tetrachlorkohlenstoff und Dichlormethan getrennt gewonnen. Die Gesamtcorticoide werden aus einer zweiten Probe von $200 \mu$ Plasma mit Dichlormethan extrahiert. Die quantitative Bestimmung der drei Corticoidfraktionen erfolgt mit Hilfe der kompetitiven Proteinbindungsmethode. Die Wiederfindung an 11-Desoxycortisol in der 11-Desoxycorticoidfraktion beträgt 57\%, die von Cortisol in der 11-Hydroxycorticoidfraktion 95\%. Der Abfall der 11-Hydroxycorticoide als Maß der Metopironwirkung und der Anstieg der 11-Desoxycorticoide im Verlauf von 8 Stunden als Parameter eines intakten oder gestörten Funktionsablaufes von Hypothalamus-Hypophyse-Nebennierenrinde werden demonstriert.
\end{abstract}

\section{A simplified metyrapone test with simultaneous determination of plasma 11-bydroxy-and 11-desoxycorticosteroids}

A simple method for estimating 11-hydroxycorticosteroids, 11-desoxycorticosteroids and total corticosteroids in plasma after the application of metyrapone is described. $4 \mathrm{~g}$ of metyrapone are orally administered during 2.5 hours; 11-desoxy- and 11-hydroxycorticosteroids are obtained and isolated from $200 \mu \mathrm{l}$ plasma by means of a "two-step-extraction" by $\mathrm{CCl}_{4}$ and $\mathrm{CH}_{2} \mathrm{Cl}_{2}$. Total corticosteroids are extracted from a second sample of $200 \mu \mathrm{l}$ plasma with $\mathrm{CH}_{2} \mathrm{Cl}_{2}$. Competitive protein binding radioassay is used to measure the steroids. The recovery of 11-desoxycortisol in the fraction of 11-desoxycorticosteroids amounts to $57 \%$, the recovery of cortisol in the fraction of 11 -hydroxycorticosteroids is $95 \%$. The sum of both corresponds to the estimated amounts of total corticosteroids. Precision, sensitivity and accuracy of the method are described.

Der Metopirontest hat seit seiner Einführung durch LIDDLE (1) Eingang in die klinische Routinediagnostik zur Überprüfung der Funktion der HypothalamusHypophysen-Nebennierenrinden-Achse gefunden (2). Durch Metopiron (2-Methyl-1,2-di-3-pyridyl-1-propanon; SU-4885; Metyrapone; Metopiron-CIBA) kommt es zu einer Hemmung der mitochondrialen Steroid$11 \beta$-Hydroxylase (1.14.1.6) der Nebennierentinde und damit zum Abfall des Plasmacortisolspiegels. Bei intakter Hypothalamus-Hypophysen-NebennierenrindenFunktion führt eine Senkung des.Cortisolspiegels zu einer gesteigerten ACTH-Ausschüttung der Hypophyse. Die durch ACTH stimulierte (3), in ihrer Cortisol-Produktion jedoch blockierte Nebennierentinde bildet vermehrt 11-Desoxycortisol (Substanz S; Cortexolon) als Vorstufe des Cortisols (4) und 11-Desoxycorticosteron (DOC; Cortexon) als Precursor von Corticosteron.

Die Metopirongabe erfolgt entweder intravenös oder aber, wie in Deutschland üblich, oral. In der Regel werden über ein oder zwei Tage verteilt mehrere Gramm Metopiron appliziert und im 24- oder 48Stdn.-Urin die vermehrte Steroidausscheidung durch Messung der reduzierenden, ketogenen, Porter-Silber chromogenen oder unpolaren Porter-Silberchromogenen Steroide (5) bestimmt. Je nach Autor und Meßmethode ist bei positivem Ausfall des Metopirontestes ein Anstieg um 100 bis über $300 \%$ zu erwarten (6).

1) Abt. Klin. Endokrinologie, Dept. Innere Medizin.
Die Anzahl der Meßmethoden und die Vielzahl ihrer Interpretationen (6) weist auf die mangelnde Spezifität dieser Gruppenbestimmungen für den Metopirontest hin. Außerdem wird im allgemeinen nur die Gesamtausscheidung von Metaboliten erfaßt, nicht aber die Kinetik der aktuellen Steroidkonzentrationen, deren Messung besonders bei Testversuchen von der Art des Metopirontestes notwendig wäre. Kommt es z. B. nach Metopirongaben nur zu einem unzureichenden Cortisolabfall im Plasma und damit zu einem ungenügenden Hypophysenreiz, wie es bei hohem Östrogenspiegel (7) und nach Diphenylhydantoin-Gaben (8) beschrieben wird, erhält man bei den oben erwähnten Messungen der Metaboliten im Urin falsch negative, pathologische Ergebnisse.

Klinisch zweifelsfreie Aussagen können somit nur dann gemacht werden, wenn durch genügend spezifische Methoden 1. der zum Hypophysenreiz führende Cortisolabfall und 2. der Anstieg von 11-Desoxycorticoiden im Plasma direkt bestimmt werden.

Grundlage des von uns angewandten Nachweises von Corticoiden im Plasma ist die von MaYes und Nugent (9) angegebene Bestimmung mittels der kompetitiven Proteinbindung mit mehreren eigenen und von LECLERCQ und Mitarbeitern (10) angegebenen Modifikationen.

Die Affinität von Transcortin, einem $\alpha_{1}$-Glykoproteid, zu Cortisol, dem hauptsächlich im Plasma vorkommenden Corticoid, ist so groß, daß eine Reinigung des 
Plasmaextraktes von nicht-corticoiden Steroiden entfallen kann.

Eine Extraktion der 11-Desoxycorticoid-Metaboliten im Urin führten HENkE und Mitarbeiter (11) nach den Untersuchungen von SILBER und Busch (12) mit Hilfe von $\mathrm{CCl}_{4}$ durch. MuRpHY und Mitarbeiter (13) wandten dieses Verfahren erstmalig auf Plasmaproben an. In Fortsetzung dieser Arbeiten möchten wir hier eine schnell und einfach durchzuführende Methode der Auswertung des Metopirontestes aus Plasmaproben beschreiben, bei der aus $200 \mu \mathrm{l}$ Plasma 11-Desoxycorticoide und 11-Hydroxycorticoide getrennt extrahiert und einzeln mit Hilfe der kompetitiven Proteinbindungsmethode nachgewiesen werden.

$\mathrm{Da}$ mit $\mathrm{CCl}_{4}$ sämtliche 11-Desoxycorticoide (s. Spezifität in "Zuverlässigkeitskriterien der Methode"), deren weit überwiegender Vertreter unter den $\mathrm{Be}$ dingungen eines Metopirontestes 11-Desoxycortisol ist, extrahiert werden, sprechen wir aus Gründen der Genauigkeit statt von 11-Desoxycortisol von den „11-Desoxycorticoiden“, sagen aus dem gleichen Grunde statt Cortisol „11-Hydroxycorticoide“ und bezeichnen die gemeinsam extrahierten Corticoide als „Gesamtcorticoide".

Unter physiologischen Bedingungen enthält die Fraktion der Gesamtcorticoide zu über $90 \%$ Cortisol, so daß dieses Verfahren in der klinischen Routinediagnostik mit ausreichender Genauigkeit zur Bestimmung des Plasmacortisolspiegels herangezogen werden kann.

\section{Methodik}

Durchführung des Metopirontestes

Metopiron wird über 2,5 Stdn. oral verabreicht (14), und zwar gibt man um $8.00 \mathrm{Uhr}$ sechs Kapseln, dann bis $10.30 \mathrm{Uhr}$ alle 30 Min. zwei Kapseln (Gesamtmenge: $4 \mathrm{~g}$ ).

Etwa $5 \mathrm{ml}$ heparinisiertes Blut werden um $8.00 \mathrm{Uhr}$ vor Einnahme des Metopirons und dann mehrfach über den Tag verteilt bis $19.00 \mathrm{Uhr}$ abends (s. Abb. 4) entnommen, sofort zentrifugiert und bei $-20^{\circ}$ bis zur Aufarbeitung und Bestimmung aufbewahrt.

\section{Auswertung des Metopirontestes \\ Reagenzien und Geräte}

Cortisol- $\left[1,2-{ }^{3} \mathrm{H}\right]$ des Radiochemical Centre, Amersham, England, mit einer spezifischen Aktivität von $43 \mathrm{C} / \mathrm{mMol}$ wird in einer Konzentration von $0,02 \mu \dot{\mathrm{C}} / \mathrm{ml}(=0,17 \mathrm{ng} / \mathrm{ml})$ in Benzol: absol. Äthanol $(9: 1, v / v)$ bei $4^{\circ}$ aufbewahrt und ohne weitere Vorreinigung verwendet. Cortisol und 11-Desoxycortisol (Merck, Darmstadt) werden in absol. Äthanol $(1 \mu \mathrm{g} / \mathrm{ml})$ bei $4^{\circ} \mathrm{im} \mathrm{Kühlschrank}$ gelagert. Die Lösungsmittel Tetrachlorkohlenstoff, Dichlormethan, Methanol, Äthanol, Toluol und Dioxan, alle vom Reinheitsgrad p. a. (Merck, Darmstadt) werden ohne Redestillation verwendet. Naphthalin; PPO, POPOP, Aktivkohle, $\mathrm{Na}_{2} \mathrm{HPO}_{4}$ und $\mathrm{KH}_{2} \mathrm{PO}_{4}$ p. a. beziehen wir von Merck, Darmstadt, und Dextran 80 von Pharmacia, Uppsala, Schweden. HamiltonMikrospritzen $(0-10$ und $0=50 \mu \mathrm{l})$ wurden von Micromesure N. V., Den Haag, Niederlande und eine Ultra-Asept-Spritze (10 ml mit Filtrationsvorsatz, Typ SM 16214, Vorfilter aus Glasfaser, Filter mit $0,2 \mu \mathrm{m}$ Porengröße) von $\mathrm{Fa}$. Sartorius, Götțingen, geliefert. Außerdem werden eine Eppendorf-Mikroliter-Pipette $(500 \mu \mathrm{l})$, eine Bürette $(5 \mathrm{ml}$, Graduierung $0,02 \mathrm{ml})$, Zentrifugengläschen (10 ml, Schliff NS 19) und selbst hergestellte konisch zulaufende Zentrifugengläser (genannt „Spitzgläschen“, lichte Weite $7 \mathrm{~mm}$, Länge $7 \mathrm{~cm}$ ) benötigt.
Zur Bestimmung der Plasmacorticoide müssen folgende Lösungen hergestellt werden:

\section{Transcortinlösung:}

$15 \mu \mathrm{l}$ der ${ }^{3} \mathrm{H}$-Cortisolstammlösung werden in einen Erlenmeyerkolben $(100 \mathrm{ml})$ pipettiert und unter Stickstoff cingedampft. Der Trockenrückstand wird sofort in $39 \mathrm{ml}$ Phosphatpuffer $\left(0,07_{\mathrm{M}}\right.$; pH 7,4) gelöst. Dann wird $1 \mathrm{ml}$ transcortinhaltiges Plasma zupipetticrt und die Lösung gut durchgemischt. Ohne nennenswerten Abfall ihrer Bindungsaffinität kann dicsc Lösung bei $4^{\circ}$ mehr als eine Woche lang aufbewahrt werden.

Das transcortinhaltige Plasma wird von endokrin gesunden, unbehandelten Personen unter Liqueminzusatz gewonnen. Um eine Uberhöhung des Nullwertes durch endogene Steroide zu vermeiden, kann das Plasma entweder

a) steroidfrei (15) oder

b) corticoidfrei (16) gemacht werden.

a) $50 \mathrm{mg}$ Aktivkohle/ml Plasma werden in ciner Schüttclmaschine bei Raumtemperatur $30 \mathrm{Min}$. lang geschüttelt. Dann wird die Kohle mit den adsorbierten Stcroiden abzentrifugiert $(8000 \mathrm{~g}$, $30 \mathrm{Min}$.) und über dic Ultra-Asept-Spritze abfiltriert. Das șo gewonnenc Plasma ist klar, weitestgehend steroidfrei und erleidet keine Einbuße seincr-Transcortinaktivität. Nach Zugabe von radioaktivem Testosteron fand sich weniger als $1 \%$ der Radioaktivität im Filtrat wieder.

b) 12 Stdn. vor Abnahme des Blutes werden dem Patienten $2 \mathrm{mg}$ Dexamethason oral gegeben. Dadurch erfolgt ein Cortisolabfall im Plasma auf kaum meßbare Werte. Die Liganden des Transcortins werden durch Dexamethason nicht besetzt.

Das auf eine der beiden Arten gewonnene Plasma wird in Fraktionen von $2 \mathrm{ml}$ bei $-20^{\circ}$ eingefroren und erst wieder zum Gebrauch aufgetaut. Im Verlaufe von sieben Monaten konnten wir keinen Abfall der Transcortinaktivität feststellen.

\section{Kohledextransuspension:}

Sowohl eine 0,5proz. Dextranlösung als auch eine 5proz. Kohlesuspension in Phosphatpuffer $(0,07 \mathrm{M} ; \mathrm{pH} 7,4)$ können bei $4^{\circ}$ praktisch unbeschränkt aufbewahrt werden. Zur Herstellung der gebrauchsfertigen Suspension werden $1 \mathrm{ml}$ der Dextran- und $1 \mathrm{ml}$ der gut durchgeschüttelten Kohlesuspension in $18 \mathrm{ml}$ Phosphatpuffer pipettiert und vor Gebrauch $30 \mathrm{Min}$. lang mit dem Magnetrührer gerührt.

3. Szintillationslösung:

$250 \mathrm{~g}$ Naphthalin, $20 \mathrm{~g}$ PPO und $0,6 \mathrm{~g}$ POPOP werden in $2500 \mathrm{ml}$ Dioxan gelöst; danach werden $230 \mathrm{ml}$ Toluol und $230 \mathrm{ml}$ absol. Äthanol zupipettiert. $10 \mathrm{ml}$ dieses Szintillators können $1,5 \mathrm{ml}$ einer wäßr. Lösung aufnehmen. Die Radioaktivität wird im Flüssigkeits-Szintillationsspektrometer $2 \mathrm{mal} 10 \mathrm{Min}$. lang gemessen (Packard Tri-carb Szintillation Spectrometer, Modell 3380).

\section{Aufarbeitung der Proben}

Das bei dem Metopirontest gewonnene und in der Tiefkühltrube gelagerte Plasma wird zur Aufarbeitung aufgetaut. Zur Bestimmung von a) Gesamtcorticoiden und b) 11-Desoxycorticoiden und 11-Hydroxycorticoiden werden jeweils $200 \mu \mathrm{l}$ der Plasmaprobe in kleine Schliffentrifugengläser pipettiert.

a) Die Gesamtcorticoide werden einmal mit $4 \mathrm{ml}$ Dichlormethan extrahiert. Nach Phasentrennung (Zentrifugierung bei $2000 \mathrm{~g}$; 3 Min.) wird die wäßr. Phase über eine Pasteurpipette mit Hilfe einer Wasserstrahlpumpe abgesaugt. Von dem klaren Dichlormethanextrakt werden $2 \mathrm{ml}$ in Spitzgläschen pipettiert und unter Stickstoff oder gereinigter Luft bei $40^{\circ}$ (Wasserbad) eingedampft. b) Die Extraktion der 11-Desoxycorticoide erfolgt mit $4 \mathrm{ml}$ Tetrach'orkohlenstoff. Die Zugabe des Tetrachlorkohlenstoffes sollte wegen der Lebertoxizität des Lösungsmittels stets unter dem Abzug mit einer Burette durchgeführt werden. Nach Phasentrennung $(2000 \mathrm{~g} ; 5 \mathrm{Min}$.) werden vorsichtig $3 \mathrm{ml}$ Tetrachlorkohlenstoff entnommen, in ein Spitzgläschen pipettiert und bei $40^{\circ}$ Wasserbadtemperatur unter gereinigter Luft eingedampft. 
Zur Extraktion der 11-Hydroxycorticoide werden anschließend $3 \mathrm{ml}$ Dichlormethan $\mathrm{zu}$ der verbleibenden Restflüssigkeit pipettiert, die sich aus $1 \mathrm{ml}$ Tetrachlorkohlenstoff und $200 \mu \mathrm{l}$ Plasma zusammensetzt. Das weiterc Aufarbeitungsverfahren entspricht dem Arbeitsgang, wie er bei den Gesamtcorticoiden beschrieben wird.

Das Extraktionsverfahren muß zwecks Reproduzierbarkeit streng standardisiert werden; so extrahieren wir durch 80 maliges gleichmäßiges Umschwenken der Schliffzentrifugengläser mit der Hand.

Zur Bestimmung der Wiederfindung bei den beschriebenen Extraktionsschritten werden jedesmal, zumindest aber bei Anbrechen einer neuen Lösungsmittellieferung, folgende Proben in Doppelbestimmung mitextrahiert und gemessen: $20 \mathrm{ng}$ Cortisol, $20 \mathrm{ng}$ 11-Desoxycortisol und $20 \mathrm{ng}$ Cortisol plus $20 \mathrm{ng}$ 11-Desoxycortisol, gelöst in jeweils $200 \mu \mathrm{l}$ steroidfreiem Plasma. Zwei Proben mit steroidfreiem Plasma $\left(\mathrm{L}_{1}\right.$ und $\mathrm{L}_{2}$ ) dienen als Leerwerte.

Zur Aufstellung der Eichkurven werden aus den Stammlösungen steigende Mengen von 11-Desoxycortisol und Cortisol $(0 ; 2,5$; $5 ; 7,5 ; 10 ; 15 ; 20 ; 30 ; 40 \mathrm{ng})$ in Spitzgläschen pipettiert und eingedampft (s. Abb. 1a).

\section{Kompetitive Proteinbindung}

In zwei mit S bezeichnete Spitzgläschen (100\%-Werte) und zu den Trockenrückständen der Proben aus den verschiedenen Extraktionen, den Wiederfindungsversuchen und den Eichkurvenbestimmungen werden $1 \mathrm{ml}$ der Transcortinlösung pipettiert. Alle Glāschen werden mit.Parafilm verschlossen und auf dem Vortexmixer $10 \mathrm{Sek}$. durchgeschüttelt. Dann stellt man die Gläschen genau $5 \mathrm{Min}$. in ein Wasserbad $\left(45^{\circ}\right)$ und anschließend etwa $30 \mathrm{Min}$. in den Kühlschrank. Die daraufhin folgende Trennung der transcortingebundenen von der nichtgebundenen Steroidfraktion wird mittels Adsorption des nicht gebundenen Anteils an dextranbeschichtete Aktivkohle durchgeführt; dieser Vorgang muß zeitgenau ablaufen, so daß man am besten nur so viel Spitzgläschen dem Kühlschrank entnimmt, wie ohne Verzug verarbeitet werden können. Zur Trennung der beiden Fraktionen werden $0,5 \mathrm{ml}$ des Kohledextrangemisches, das durch einen Magnetrührer in einer gleichmäßigen Suspension gehalten wird, mit einer Eppendorf-Mikroliter-Pipette in jedes eisgekühlte Spitzgläschen transferiert. Dadurch, daß man die KobleDextran-Suspension möglichst fest in die Transcortinlösung jedes Gläschens hineinpreßt, wird eine ausreichende Durchmischung erreicht. In die beiden mit $\mathrm{S}$ bezeichneten 100\%-Werte werden $0,5 \mathrm{ml}$ der Pufferlösung und zu zwei mit $\mathrm{L}_{3}$ und $\mathrm{L}_{4}$ beschrifteten Leerwerten $1,5 \mathrm{ml}$ Puffer pipettiert. Jede Probe verbleibt daraufhin genau $10 \mathrm{Min}$. in Eiswasser, bevor die Kohlepartikel, an die die ungebundenen Steroide adsorbiert sind, abzentrifugiert werden ( $2000 \mathrm{~g}$; genau $4 \mathrm{Min}$.). Jeweils $1 \mathrm{ml}$ des klaren Ưberstandes wird in Szintillationszählgläschen überführt; bei gleichförmig hergestellten Spitzgläschen kann auch der gesamte Uberstand in die Zählgläschen dekantiert werden (10). Nach Zugabe von $10 \mathrm{ml}$ Szintillationslösung wird die Radioaktivität jeder Probe im Flüssigkeitsszintillationszähler bestimmt (2 mal 10 Min., „preset counts“ 10000 Imp./Min.).

\section{Bestimmung der Steroidmenge aus der gemessenen Radioaktivität}

Die im Flüssigkeitsszintillationszähler gemessene Radioaktivität der Eichwerte, ausgedrückt in Prozent der Gesamtradioaktivität $(\mathrm{S}=100 \%)$, ergibt im Koordinatensystem als Funktion der zugegebenen Steroidmenge eine Eichkurve (Abb. 1a), während die Kehrwerte der bei den Eichwerten gemessenen Radioaktivität als Funktion der Steroidmenge eine Gerade ergeben (Abb. 1b). Aus den auf gleiche Weise umgerechneten Werten der Proben kann die entsprechende Steroidmenge in den Eichkurven abgelesen werden.

Bei Bestimmung der 11-Desoxycorticoide wird die 11-Desóxycortisol-Eichgerade und bei Bestimmung der Corticoide die

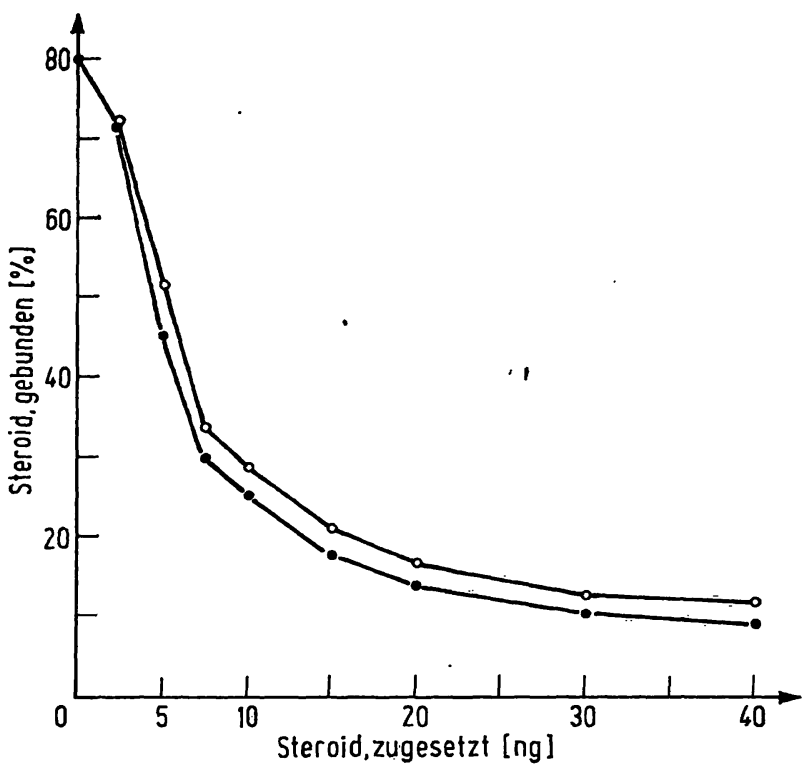

Abb. 1 a

Eichkurven für Cortisol $(\bullet-\bullet)$ und 11-Desoxycortisol $(\circ-0)$; Prozent gebundenes Steroid, aufgetragen gegen die eingesetzte. Steroidmenge

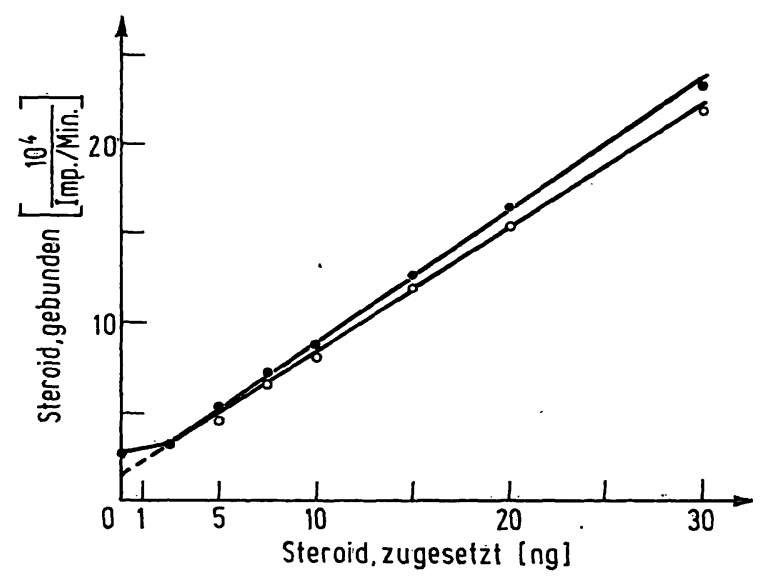

Abb. $1 \mathrm{~b}$

Eichkurven für Cortisol und 11-Desoxycortisol; gebundenes Steroid (y), ausgedrückt als Kehrwert der gemessenen Radioaktivität (Imp./Min.), aufgetragen gegen die zugegebene Steroidmenge (x). Der Anstieg der Geraden (m) ergibt sich aus $\mathrm{y}=\mathrm{mx}+\mathrm{b}$ und beträgt hier für die Cortisol-Eichgerade 0,75 und für die 11 -DesoxycortisolEichgerade 0,7

Cortisol-Eichgerade herangezogen. Aus den Wiederfindungsversuchen (Tab. 1) ist jedoch ersichtlich, daß die Extraktion der 11-Desoxycorticoide mit Tetrachlorkohlenstoff nicht vollständig ist und daß somit in der 11-Hydroxycorticoidfraktion und auch in der Gesamtcorticoidfraktion 11-Desoxycorticoide vorhanden sein können. Daher muß für einen solchen Mischwert eine neue Eichgerade erstellt werden. Diese neu zu erstellende Gerade verschiebt sich bei hohem Anteil an 11-Desoxycorticoiden mehr' zur 11-Desoxycortisol-Eichgeraden hin und bei niedrigem Anteil mehr in die Nähe der Cortisol-Eichgeraden. $\mathrm{Da}$ die Menge an 11-Desoxycorticoiden aus der Tetrachlorkohlenstofffraktion bei Kenntnis der Wiederfindung bestimmt werden kann, ist auch die 11-Desoxycorticoidmenge in der 11-Hydroxycorticoid- und Gesamtcorticoidfraktion bekannt. Daraus kann der prozentuale Anteil an 11-Desoxycorticoiden in der Gesamt- und in der 11Hydroxycorticoidfraktion mit Hilfe einer der beiden Eichgeraden bestimmt werden. Dieser prozentualen Verteilung entsprechend liegt die neue Eichgerade zwischen der Cortisol- und der 11-Desoxycortisoleichgeraden. Der daraus errechnete Wert für die Summe der beiden Steroide ist bei der geringen Differenz in der Steigung der beiden Geraden befriedigend genau. 
Fragen Sie bei SIGMA nach

\section{Ein-Reagenz- und Zwei-Reagenz-Systemen für die Bestimmung von SGOT/SGPT}

(Aspartat- + Alanin-Transaminase)

mit dem optischen Test in Serum oder anderen biologischen Flüssigkeiten bei $340 \mathrm{~nm}$

\section{„Stat", manuelle oder automatisierte kinetische Verfahren}

Ein-Reagenz-System - Sigma Technical Bulletin Nr. 55-UV.

Ein schnelles, einfaches Verfahren für Routine- oder "Stat"bestimmungen von Hand oder mit automatisierten Systemen wie GEMSAEC, Centrifichem, Rotochem u. a., die mit nur einem Reagenz arbeiłen. Sie brauchen nur Wasser und Serum hinzuzufügen und dann die Extinktion abzulesen; erhältlich sind entsprechend Ihren Erfordernissen Reagenzampullen für eine, fünf oder zehn Bestimmungen.

Das Sigma Technical Bulletin Nr. 55-UV erhalten Sie auf Anfrage gratis.

Bitte, bestellen Sie:

\begin{tabular}{|c|c|c|c|c|c|c|c|c|}
\hline $\begin{array}{l}\text { Lager- } \\
\text { nummer }\end{array}$ & $\begin{array}{l}\text { Reagenz- } \\
\text { ampullen } \\
\text { für SGOT }\end{array}$ & $\begin{array}{c}\text { Handels- } \\
\text { größen }\end{array}$ & $\begin{array}{r}\text { maxima } \\
\text { mögliche } \\
\text { Bestimmung }\end{array}$ & Preis & $\begin{array}{l}\text { Lager- } \\
\text { nummer }\end{array}$ & $\begin{array}{l}\text { Reagenz- } \\
\text { r ampullen } \\
\text { für SGPT }\end{array}$ & $\begin{array}{l}\text { maximal } \\
\text { mögliche } \\
\text { Bestimmunger }\end{array}$ & Preis \\
\hline $55-1$ & $\begin{array}{l}\text { für } 1 \text { Bestim- } \\
\text { mung }\end{array}$ & 10 Ampullen & 10 & $\$ 5,00$ & $55-1 P$ & $\begin{array}{l}\text { für } 1 \text { Bestim- } 10 \text { Ampullen } \\
\text { mung }\end{array}$ & 10 & $\$ 5,00$ \\
\hline $\begin{array}{l}55-5 \\
55-10\end{array}$ & $\begin{array}{l}\text { für } 5 \text { Bestim- } \\
\text { mungen } \\
\text { für } 10 \mathrm{Be}- \\
\text { stimmungen }\end{array}$ & $\begin{array}{l}5 \text { Ampullen } \\
10 \text { Ampullen } \\
5 \text { Ampullen } \\
10 \text { Ampullen }\end{array}$ & $\begin{aligned} & 5 \times 5 \\
& 10 \times 5 \\
& 5 \times 10 \\
& 10 \times 10\end{aligned}$ & $\begin{array}{l}\$ 7,50 \\
\$ 14,85 \\
\$ 12,50 \\
\$ 24,85\end{array}$ & $\begin{array}{ll}55-5 P \\
55-10 P\end{array}$ & $\begin{array}{l}\text { für } 5 \text { Bestim- } 5 \text { Ampullen } \\
\text { mungen } 10 \text { Ampullen } \\
\text { für } 10 \mathrm{Be}- \\
\text { stimmungen } 10 \text { Ampullen } \\
\text { Ampullen }\end{array}$ & $\begin{aligned} & 5 \times 5 \\
& 10 \times 5 \\
& 5 \times 10 \\
& 10 \times 10\end{aligned}$ & $\begin{array}{l}\$ 8,00 \\
\$ 15,50 \\
\$ 14,00 \\
\$ 27,00\end{array}$ \\
\hline
\end{tabular}

Zwei-Reagenz-Systeme - Sigma Technical Bulletin Nr. 155-UV.

Für automatisierte Analysengeräte, die für Zwei-Reagenz-Systeme ausgelegt sind, wie z. B. LKB Reaction Rate Analyzer und der Beckman DSA-560 und 564-B.

Kann ebenso für manuelle Verfahren und für automatisierte Analysengeräte, die mit nur einem Reagenz arbeiten, verwandt werden.

Das Sigma Technical Bulletin Nr. 155-UV erhalten Sie auf Anfrage gratis.

$\begin{array}{ccccc}\text { Bitte, bestellen Sie: } & \text { Besteck-Nr. } & \begin{array}{c}\text { Art der } \\ \text { Bestimmung }\end{array} & \begin{array}{c}\text { maximal mögliche } \\ \text { Bestimmungen* }\end{array} & \text { Preis } \\ & \text { 155-A } & \text { SGOT } & 100 & \$ 22,50 \\ & 155-B & \text { SGPT } & 100 & \$ 24,50 \\ & \text { *Bezüglich der Anzahl der Bestimmungen siehe die oben gemachten } & \text { Bemerkungen }\end{array}$

Natürlich bieten wir weiterhin das Original kolorimetrische Verfahren - erprobt in der ganzen Welt - überall imitiert, jedoch nie erreicht - an.

Nr. 505 - Sigma-Fränkel kolorimetrische Bestimmung bei $490-530 \cdot \mathrm{nm}$

Besteck-Nr.
Art der maximal Preis Bestimmung mögliche Bestimmungen

$\begin{array}{lcrr}505 & \text { SGOT } & 100 & \$ 10,00 \\ 505-M & \text { SGOT } & 25 & \$ 5,50 \\ 505-P & \text { SGPT } & 100 & \$ 10,00 \\ 505-P M & \text { SGPT } & 25 & \$ 5,50 \\ 505-O P & \text { SGOT/SGPT } & 100 & \$ 15,00\end{array}$

Ferner ist erhältlich

Nr. 410-UV - Bestimmung im optischen Test bei $340 \mathrm{~nm}$

Besteck-Nr. Art der maximal Preis Bestimmung mögliche

Bestimmungen

$\begin{array}{lcrr}410-50 & \text { SGOT } & 50 & \$ 11,55 \\ 410-100 & \text { SGOT } & 100 & \$ 22,45 \\ 410-50 P & \text { SGPT } & 50 & \$ 14,75 \\ 410-O P & \text { SGOT/SGPT } & \text { je } 50 & \$ 26,30\end{array}$

kombiniert

Sigma Technical Bulletins für die oben aufgeführten Methoden sind auf Anfrage gratis erhältlich.

Alle Preise einschließlich normaler Verșandgebühr zu lhnen überall hin.in die Welt.

Es ist ein Vergnügen, Geschäfte mit Sigma zu machen

Bestellen Sie direkt - R-Gespräch von überall her in der Welt Tagsüber von Haus zu Haus, 314-771-5750. Nachts von Person zu Person, Dan Broida, 314-993-6418 TWX (Fernschreiber) Tag und Nacht: Rückantwort 910-761-0593

Sigma-Reagenzien sind in der ganzen Welt durch den Fachhandel oder direkt aus St. Louis beziehbar.

Telegramme: SIGMACHEM, St. Louis, Missouri

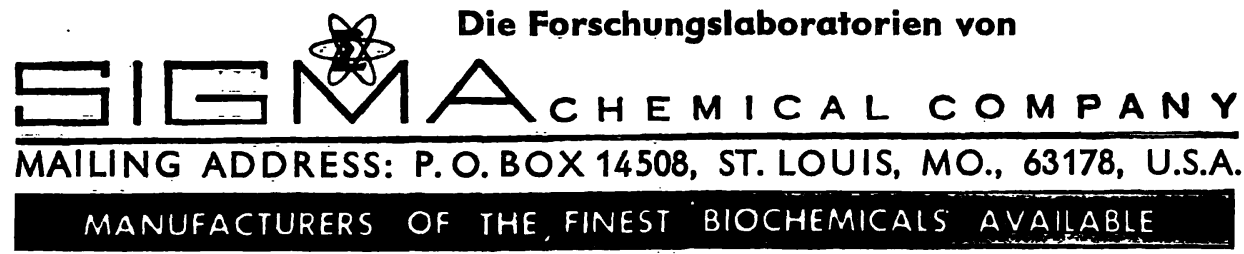

Vertreten durch

SIGMA LONDON Chem. Co. Ltd. - 12, Lettice St., London, S. W. 6., England Telephone: 01-736-5823 (Reverse Charges)

SIGMA ISRAEL Chem. Co. Ltd. @ 28 Kaf-Gimel St., Givataim, Israel Telephone: (03) 760654 (Reverse Charges) 


\section{Klinische Chemie}

Theorie und Praxis

3., überarbeitete Auflage

R. Richterich (Bern)

XXII + 599 Seiten, 95 Abbildungen, 90 Tabellen, 1 Farbtafel, 1971 SFr. 98. - I US $\$ 29.50$ / DM 89, - / £ 10.30

Teil A Einleitung: Allgemeines - Mass-Einheiten - Einführung in die Statistik - Normalwerte Zuverlässigkeit von Labor-Methoden und Qualitäts-Kontrolle - Labor-Räume und Labor-Geräte - Chemikalien - Blut-Entnahme - Urin-Sammlung

Teil B Bestimmungs-Methoden: Allgemeines - Trennungs-Methoden - Gravimetrie - Volumetrie - Optische Mess-Methoden - Enzym-Bestimmungen

Teil C Stoffwechsel-Untersuchungen: Allgemeines - Sạure-Basen- und Elektrolyt-Sfoffwechsel Spuren-Elemente - Energie-Stoffwechsel - Kohlenhydrat-Stoffwechsel - StickstoffStoffwechsel - Lipid-Stoffwechsel - Nukleinsäure-Stoffwechsel - Pharmakologie, Toxikologie

Teil D Organ-spezifische Untersuchungen: Einleitung - Knochen - Skelettmuskel - Herz Hämatopoietisches System - Magen-Darm-Trakt - Pankreas und Speicheldrüsen Leber-Krankheiten - Endokrine Drüsen - Nervensystem - Männliche Genitạlorgane - Nieren

Anhang - Reklameteil

Bereits 6 Jahre nach ihrem ersten Erscheinen wurde eine 3., erweiterte Auflage der „Klinischen Chemie" notwendig. Dies spricht für die Bedeutung dieses Buches, in dem sowohl Theorie als auch Praxis dieses immer wichtiger werdenden Gebietes ausführlich dargestellt sind. Die überarbeitete Auflage ist etwa 50 Seiten umfangreicher geworden und enthält mehrere neve theoretische und methodische Kapitel. Besonders zu begrüßen ist die ausführliche Darstellung der Methoden zur Analyse des Säure-BasenHaushaltes.

\section{S. Karger}

Basel - München - Paris · London - New York - Sydney 
Tab. 1 a

Ergebnisse aus Wiederfindungsversuchen; die Zahlen bedeuten Wiederfindung \pm Standardabweichung in Prozent der zugegebenen Steroidmenge

\begin{tabular}{|c|c|c|c|c|}
\hline \multicolumn{5}{|c|}{$100 \mu l$ steroidfreiem Plasma wurde zugegeben } \\
\hline $\begin{array}{l}\text { Wiederfindung in der } \\
\text { Fraktion der }\end{array}$ & $5 \mathrm{ng}$ & $10 \mathrm{ng}$ & $5 \mathrm{ng}^{11-D}$ & $\stackrel{\text { ortisol }}{10} \mathrm{ng}$ \\
\hline $\begin{array}{l}\text { 11-Desoxycorticoide } \\
\text { 11-Hydroxycorticoide } \\
\text { Gesamtcorticoide }\end{array}$ & $\begin{array}{r}2,8 \pm 1,5 \% * \\
92,0 \pm 2,4 \% * \\
101,0 \pm 1,9 \%\end{array}$ & $\begin{array}{r}2,2 \pm 1,4 \% \\
94,5 \pm 2,3 \% \\
97,0 \pm 2,7 \%\end{array}$ & $\begin{array}{l}59,3 \pm 3,0 \% \\
42,1 \pm 2,9 \% \\
99,5 \pm 1,6 \%\end{array}$ & $\begin{array}{l}56,7 \pm 2,4 \% * \\
40,1 \pm 2,6 \% * \\
99,8 \pm 1,3 \%\end{array}$ \\
\hline
\end{tabular}

$(n: 10 ; *) n: 5)$

Tab. $1 \mathrm{~b}$

Ergebnisse aus Wiederfindungsversuchen; gemäß den Ergebnissen in der Tabelle 1 a wurden die Werte um den Anteil an mitextrahierten nicht der Fraktion zugehörenden Steroiden korrigiert. Um den physiologischen Gegebenheiten möglichst nahe zu kommen, wurden Cortisol und 11-Desoxycortisol steroidfreiem Plasma zugegeben ( $\mathrm{n}: 9)$

\begin{tabular}{lcc}
\hline $\begin{array}{c}\text { Wiederfindung in der } \\
\text { Fraktion der }\end{array}$ & $\begin{array}{c}100 \mu l \text { steroidfreiem Plasma wurde } \\
\text { ein Gemisch von 11-Desoxycortisol } \\
\text { und Cortisol zu gleichen Teilen } \\
\text { zugegeben }\end{array}$ \\
& $5 \mathrm{ng}$ & $10 \mathrm{ng}$ \\
\hline 11-Desoxycorticoide & $59,2 \pm 2,4 \%$ & $58,2 \pm 1,1 \%$ \\
11-Hydroxycorticoide & $97,4 \pm 2,3 \%$ & $96,2 \pm 2,3 \%$ \\
Gesamtcorticoide & $96,5 \pm 3,4 \%$ & $95,4 \pm 3,7 \%$ \\
\hline
\end{tabular}

Die Berecbnung der 11-Desoxycorticoide in $100 \mathrm{ml}$ Plasma wird nach folgender Beziehung durchgeführt:

ng 11-Desoxycorticoid/Meßansatz $\cdot \mathrm{V} \cdot \mathrm{W} \cdot 10000=\mu \mathrm{g} / \mathrm{l}$ Plasma. Dabei wird ng/Meßansatz aus der gemessenen Radioaktivität mit Hilfe der Eichgeraden bestimmt; der Volumenfaktor $(V)$ ist 0,67 bezogen auf $100 \mu \mathrm{l}$ extrahierten Plasmas, und die Wiederfindung (W) liegt je nach Lösungsmittellieferung zwischen 55 und $60 \%$ (Tab. 1 ).

Die Menge an Gesamtcorticoiden kann direkt aus der Eichgeraden bestimmt werden, da der Volumenfaktor so gewählt wurde, da B der 10 fache Wert ng Gesamtcorticoid/Meßansatz dem Wert $\mu \mathrm{g} / 1$ Plasma entspricht. Die Wiederfindung für das Extraktionsverfahren liegt nahe bei $100 \%$.

Die 11-Hydroxycorticoidmenge wird nach folgender Beziehung berechnet: ng 11-Hydroxycorticoid/Meßansatz $\cdot \mathrm{W} \cdot 10000-\mathrm{F}=\mu \mathrm{g} / 1$ Plasma. ng 11-Hydroxycorticoid/Meßansatz wird mit Hilfe der Eichgeraden aus der gemessenen Radioaktivität bestimmt. Die Wiederfindung (W) liegt bei $95 \%$ (Tab. 1). F entspricht den in der 11-Hydroxycorticoidfraktion mitextrahierten 11-Desoxycorticoiden, die sich zusammensetzen aus den nach Tetrachlorkohlenstoff-Extraktion in der wäßr. Phase verbleibenden 11-Desoxycorticoiden, die nach den Wiederfindungsversuchen $40-45 \%$ der 11-Desoxycorticoide betragen (Tab. 1) und den 11-Desoxycorticoiden des einen Milliliters Tetrachlorkohlenstoff aus der 11-Desoxycorticoidfraktion, das bei unserem Aufarbeitungsverfahren in der 11-Hydroxycorticoidfraktion verbleibt. Beträgt z. B. die Wiederfindung der 11-Desoxycorticoide in der 11Desoxycorticoidfraktion $60 \%$, dann sind in dem verbleibenden Millimeter Tetrachlorkohlenstoff $15 \%$ der 11 -Desoxycorticoide enthalten.

Für den klinischen Bedarf wird die Bestimmung der 11-Desoxycorticoide und der Gesamtcorticoide genügen, aus deren Differenz sich die Menge an 11-Hydroxycorticoiden 'ergibt (cf. Zuverlässigkeitskriterien).

\section{Zuverlässigkeitskriterien der Methode}

\section{Spezifität}

Außer 11-Desoxycortisol und Cortisol können bei der hier beschriebenen Bestimmungsmethode noch andere Steroide miterfaßt werden. Unter Normalbedingungen gehört dazu bei der Messung der Gesamtcorticoide das Corticosteron, das durch Dichlormethan mitextrahiert wird und bis $\mathrm{zu} 10 \%$ der als Cortisol gemessenen Fraktion ausmachen kann. Daß trotz dieser Einschränkung die Gesamtcorticoide als "Cortisol" angesprochen werden können, zeigen Versuche, Cortisol nach chromatographischer Reinigung mit Hilfe der kompetitiven Proteinbindungsmethode oder mittels der Doppelisotopenderivat-Verdünnungsmethode spezifisch nachzuweisen, wo sich im Normalbereich praktisch keine Unterschiede für den aktuellen Plasmacortisolspiegel finden (17). Anders ist es bei der Beurteilung des Metopirontestes, bei dem die Gesamtcorticoide nur ein Maß der Gesamtsituation für corticoide Steroide im Plasma darstellen. Die dadurch notwendige, hier beschriebene Fraktionierung in 11-Desoxy- und 11-Hydroxycorticoide erfaßt in der 11-Hydroxycorticoidfraktion praktisch ausschließlich Cortisol, in der 11-Desoxycorticoidfraktion neben dem weit überwiegenden 11-Desoxycortisol auch 11-Desoxycorticosteron (18), und - nicht ins Gewicht fallend - auch Progesteron und 17Hydroxyprogesteron (19).

\section{Richtigkeit}

Die Richtigkeit ergibt sich aus Wiederfindungsversuchen mit authentischem Cortisol und 11-Desoxycortisol, die in verschiedenen Konzentrationen steroidfreiem Plasma (Tab. 1a) zugesetzt werden. Die Wiederfindung bei Bestimmung der Gesamtcorticoide ist mit $100 \%$ sowohl bei Extraktion mit Dichlormethan als auch mit Dichlormethan/Tetrachlorkohlenstoff $(3: 1)$ für beide gleich. Cortisol wird mit Tetrachlorkohlenstoff $z u$ etwa $2 \%$ extrahiert, während die Extraktion des 11-Desoxycortisols durch Tetrachlorkohlenstoff 55 bis $60 \%$ beträgt (Tab. 1a). Durch die nach Tetrachlorkohlenstoffextraktion folgende Dichlormethanextraktion werden sämtliche in der wäßr. Phase verbliebenen Steroide gewonnen, so daß die Summe der Steroide in den beiden Fraktionen erwartungsgemä $\beta$ der Menge an Gesamtsteroid entspricht. $\mathrm{Da} \beta$ unser Verfahren nicht nur bei der Zugabe von Einzelsteroiden reproduzierbare Werte liefert, zeigen die Ergebnisse nach Extraktion eines $\mathrm{Ge}$ misches von gleichen Teilen Cortisol und 11-Desoxycortisol (Tab. 1b). Dabei finden sich $59,2 \%$ bei Zugabe von 5 ng Steroid und $58,4 \%$ bei 10 ng Steroidzugabe in der 11-Desoxycorticoidfraktion wieder, während $97,4 \%$ (bei $5 \mathrm{ng}$ ) und $96,2 \%$ (bei $10 \mathrm{ng}$ ) des 


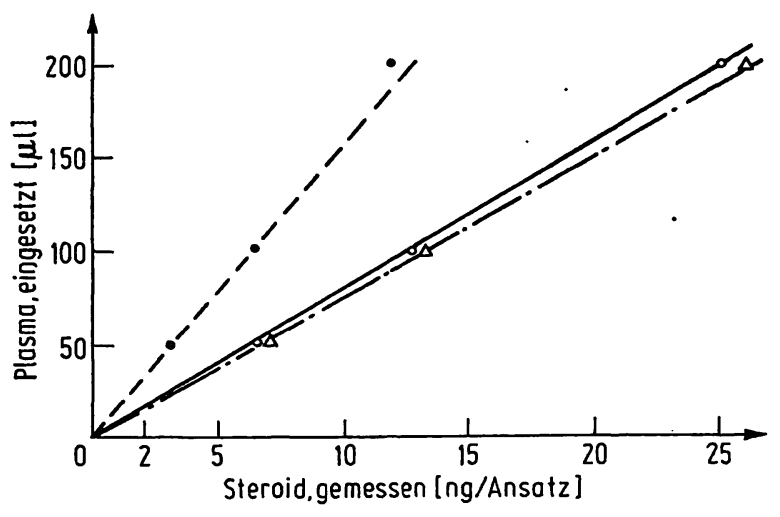

Abb. 2

Bestimmung der 11-Desoxycorticoid-, der 11-Hydroxycorticoid- und der Gesamtcorticoidfraktion aus ansteigenden Plasmamengen; jeder Punkt der drei Kurven entspricht dem Mittelwert aus fünf Einzelbestimmungen. 11 -Hydroxycorticoide; - - 11-Desoxycorticoide (ng $\times 10^{-1} /$ Ansatz); - - - Gesamtcorticoide

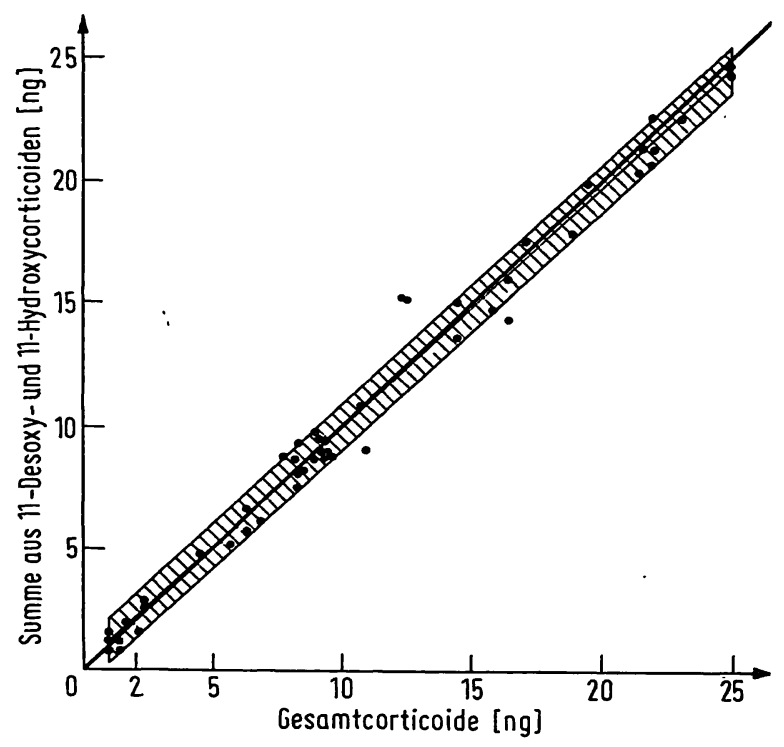

Abb. 3

Regressionsgerade zur Überprüfung der Richtigkeit des Extraktionsund Bestimmungsverfahrens; zur Überprüfung der Richtigkeit wurde bei 40 Proben die in der Fraktion der Gesamtcorticoide gemessene Steroidmenge (Istwert) der Summe aus den gemessenen 11-Desoxyund 11-Hydroxycorticoiden (Sollwert) gegenübergestellt. Die Regressionsgleichung obiger Kurve lautet:

$y=0,22+0,97 x ; r: 0,985 ;$ die Streuung beträgt Sy $\cdot x: 0,97$

Cortisols nach anschließender Dichlormethanextraktion gemessen werden können. Die Extraktion beider Steroide in der Fraktion der Gesamtsteroide liegt bei $96 \%$. Werden die Steroide aus steigenden Volumina eines Plasmapools extrahiert und gemessen, so ergeben sich in allen drei Fraktionen lineare Beziehungen zwischen Volumen und gemessener Steroidmenge (Abb. 2). Die Tatsache, daß alle drei Geraden durch den Nullpunkt des Koordinatensystems laufen, mag neben dem Kriterium für die Richtigkeit der Methode auch ein Beweis ihrer Spezifität sein (20). Zur Uberprüfung der Richtigkeit der Nachweismethode wurden außerdem bei 40 Werten von Gesamtcorticoiden, die sowohl 11-Desoxycorticoide als auch 11-Hydroxycorticoide enthielten, der direkt gemessene Wert (= Istwert) der Summe aus 11-Hydroxy- und 11-Desoxycorticoiden
(=Sollwert) gegenübergestellt. Die dabei erhaltene Regressionsgerade ist in Abbildung 3 festgehalten und lautet: $y=0,22+0,97 x ; r: 0,985$.

\section{Genauigkeit}

Zur Berechnung der Präzision wurden Mehrfachbestimmungen aus Plasmaproben vor und nach Metopironapplikation durchgeführt. Dabei fand sich bei zehnfacher Bestimmung der Corticoide aus einer Plasmaprobe vor Metopironeinnahme nach vorhergegangener Tetrachlorkohlenstoffextraktion ein Wert von $88 \pm 6,1 \mu \mathrm{g} / 1$ Plasma (VK: 6,1\%); 11-Desoxycorticoide waren nicht nachweisbar. Nach Metopiron betrug der Wert $128 \pm 4 \mu \mathrm{g}$ 11-Desoxycorticoid/1 Plasma (VK: 3,1\%).

Bei 34 Proben, die sowohl 11-Desoxycortisol als auch Cortisol enthielten, konnten nach Doppelbestimmung die in Tabelle 2 aufgeführten Ergebniśse erzielt werden.

Tab. 2

Präzision von Doppelbestimmungen in verschiedenen Konzentrationsbereichen

\begin{tabular}{|c|c|c|c|c|c|}
\hline Fraktion der & $\begin{array}{l}\text { Konzen- } \\
\text { trations- } \\
\text { Bereich } \\
\mu \mathrm{g} \text { Steroid/1 } \\
\text { Plasma }\end{array}$ & $\mathbf{n}$ & $\underset{\mu \mathrm{g} / 1}{\dot{\bar{x}}}$ & $\underset{\mu \mathrm{g} / \mathrm{l}}{\mathrm{s}}$ & $\begin{array}{r}\text { VK } \\
\%\end{array}$ \\
\hline 11-Desoxycorticoide & $\begin{array}{c}2-50 \\
51-100 \\
>100\end{array}$ & $\begin{array}{r}13 \\
12 \\
9\end{array}$ & $\begin{array}{r}22 \\
64 \\
136\end{array}$ & $\begin{array}{r}6,3 \\
6,4 \\
12,0\end{array}$ & $\begin{array}{r}28,6 \\
10,0 \\
8,9\end{array}$ \\
\hline 11-Hydroxycorticoide & $\begin{array}{c}2-50 \\
51-100 \\
>100\end{array}$ & $\begin{array}{r}15 \\
12 \\
7\end{array}$ & $\begin{array}{r}26 \\
77 \\
121\end{array}$ & $\begin{array}{l}6,0 \\
7,1 \\
8,2\end{array}$ & $\begin{array}{r}23,1 \\
9,3 \\
6,8\end{array}$ \\
\hline Gesamtcorticoide & $\begin{array}{c}2-50 \\
51-100 \\
>100\end{array}$ & $\begin{array}{r}4 \\
1.0 \\
20\end{array}$ & $\begin{array}{r}41 \\
78 \\
155\end{array}$ & $\begin{array}{r}\overline{7,4} \\
15,0\end{array}$ & $\begin{array}{l}\overline{9,5} \\
9,7\end{array}$ \\
\hline
\end{tabular}

\section{Empfindlichkeit}

Die Empfindlichkeit beträgt sowohl für 11-Desoxycortisol als auch für Cortisol 1,2 ng/Ansatz. 'Bei Versuchen, den niedrigst meßbaren Wert zu bestimmen, der signifikant unterschiedlich ist von Null, fand sich bei 11-Desoxycortisol ein Wert von $10 \mu \mathrm{g} / 1$ ( $\mathrm{t}: 12,23$; $\mathrm{p}<0,001)$ und bei Cortisol $5 \mu \mathrm{g} / \mathrm{l}(\mathrm{t}: 14,25 ; \mathrm{p}<0,001)$.

\section{Reproduzierbarkeit}

Zur Bestimmung der Reproduzierbarkeit wurden aliquote Anteile einer Probe nach Metopirongabe eingefroren ( $n: 8)$ und im Verlaufe von 5 Monaten getrennt aufgearbeitet. Die dabei gefundenen Werte waren für 11-Desoxycorticoide 80,6 $\pm 6,4 \mu \mathrm{g} / \mathrm{l}$ (VK: 7,9\%), für 11-Hydroxycorticoide 1,5 $\pm 2,1 \mu \mathrm{g} / 1$ ( $=$ unterhalb der sicheren Nachweisgrenze) und für die Gesamtcorticoide $83 \pm 7,8 \mu \mathrm{g} / 1$ (VK: 9,4\%).

\section{Diskussion}

Die hier beschriebene Methode der Bestimmung von 11-Hydroxy- und 11-Desoxycorticoiden im Plasma mit Hilfe der kompetitiven Proteinbindung nach zweistufiger fraktionierter Extraktion ist einfach und 
schnell durchzuführen. Eine gut eingearbeitete medizinisch-technische Assistentin kann an zwei Arbeitstagen bei rund 40 Plasmaproben die 11-Hydroxy-, die 11-Desoxy- und die Gesamtcorticoidfraktionen aufarbeiten und messen.

Bei standardisierter Extraktion und Bestimmung lassen sich, wie die Zuverlässigkeitskriterien ausweisen, befriedigend genaue Ergebnisse erzielen. Der gewisse Mangel an Spezifität ist bei der Beurteilung des Metopirontests außer während der Schwangerschaft mehr von theoretischer als von praktischer Bedeutung, denn die Affinität von Transcortin zu anderen Steroiden als 11-Desoxycortisol und Cortisol (in Frage kommen Corticosteron, Cortison, Progesteron und $17 \alpha-\mathrm{Hy}-$ droxyprogesteron) ist entweder bedeutend geringer oder aber die Menge dieser Steroide ist unter den Bedingungen des Metopirontestes quantitativ ohne Relevanz.

Bei Messung des Plasmacortisolwertes unter Normalbedingungen kann das zur Bestimmung der Gesamtcorticoide beschriebene Verfahren herangezogen werden. Dabei ist jedoch zu beachten, daß Corticosteron, falls es nicht vorher mit $\mathrm{CCl}_{4}$ eliminiert wurde, $\mathrm{zu}$ leicht überhöhten "Plasmacortisolspiegeln" führt. Die Empfindlichkeit der Nachweismethode ist durch gröBere Verdünnung der Transcortinlösung leicht zu steigern; die von uns gewählte Transcortinkonzentration erlaubt die sichere Bestimmung im Plasma physiologisch vorkommender Corticoidmengen. Die viel angewendete fluorometrische Bestimmungsmethode der Corticoide hat gegenüber dem Nachweis mit Hilfe der kompetitiven Proteinbindung folgende Nachteile: eine relativ große, für die Bestimmung erforderliche Plasmamenge, große Fehlermöglichkeiten durch Verunreinigung der Lösungsmittel, größerer Arbeits- und Zeitaufwand und starke Beeinflussung durch vom Patienten eingenommene Pharmaka wie Chinin, Sulfonamide, Tetracycline, Sedativa (21, 22). Nach den Untersuchungen von Murphy (23) konnte dagegen kein gebräuchliches, die Proteinbindungșreaktion becinflussendes Medikament gefunden werden.

Um sicherzugehen, da $\beta$ einmal die Resorption des Metopirons aus dem Darm ausreichend ist und zum anderen die Wirkung des Metopirons an der Nebennierenrinde in der Hemmung der Steroid-11 $\beta-\mathrm{Hy}-$ droxylase voll wirksam wird, bestimmen wir regelmäßig den Cortisolplasmaspiegel vor und zwei Stunden nach Metopironeinnahme.

Ein Beispiel für derı Verlauf der von uns bestimmten Steroidkonzentrationen im Plasma nach Metopirongabe bei einem gesunden 19jährigen Probanden ist in $\mathrm{Ab}-$ bildung 4 dargestellt. Ausgehend von einem hohen 11-Hydroxycorticoidwert (155 $\mu \mathrm{g} / \mathrm{l})$ tritt nach Gaben von Metopiron entsprechend unserem Schema innerhalb von 2 Stunden ein Abfall auf $30 \mu \mathrm{g} / \mathrm{l}$ und nach 8 Stunden auf nicht mehr meßbare Werte ein. Auf diesen Stimulus hin steigen über eine vermehrte ACTH-Ausschüttung die 11-Desoxycorticoide im Plasma an, die ab $10.00 \mathrm{Uhr}$

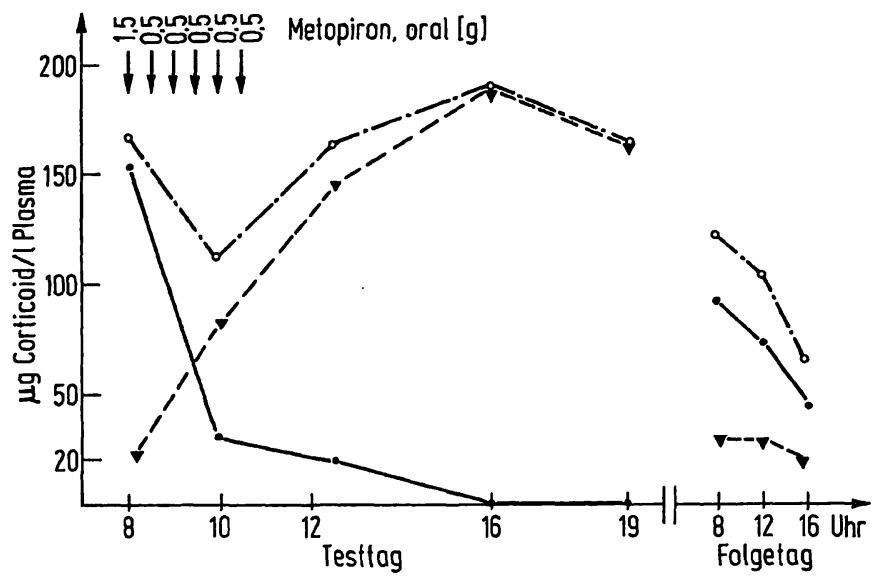

Abb. 4

11-Desoxy-, 11-Hydroxy- und Gesamtcorticoide im Plasma während eines Metopirontestes bei einem gesunden 19jährigen Probanden, normaler Verlauf



Abb. 5

11-Desoxy-, 11-Hydroxy- und Gesamtcorticoide im Plasma während eines Metopirontestes bei einem 60jährigen Patienten zwei Wochen nach Herzinfarkt; mangelnder 11-Desoxycorticoidanstieg

11-Hydroxycorticoide; --11 -Desoxycorticoide; -. - - - - Gesamtcorticoide

kontinuierlich bis zu einem Maximum gegen 16.00 Uhr zunehmen. Mit $190 \mu \mathrm{g} / 1$ 11-Desoxycorticoid wird sogar der Ausgangswert der 11-Hydroxycorticoide überschritten. Obwohl sich am folgenden Tag ein noch normales Gesamtcorticoid-Tagesprofil zeigt, ist der 11-Hydroxycorticoid-Morgenwert noch weit unter dem des Vortages und der 11-Desoxycorticoidwert deutlich erhöht. Dies könnte einmal für eine sich erschöpfende ACTH-Reserve der Hypophyse sprechen und bzw. oder für eine verlängerte, wenn auch abgeschwächte Metopironwirkung. Diese prolongierte Wirkung von Metopiron weist auf mögliche Gefahren des Metopirontestes hin. Schon bei normal ausfallendem Test ist bei unseren Versuchsbedingungen der Organismus über einige Stunden glucocorticoidfrei und damit weniger „stress-resistent", bei pathologischem Ausfall des Testes dauert dieser glucocorticoidfreie Zustand des Organismus jedoch einen ganzen Tag (Abb. 5). Diese Gefahr gilt jedoch in viel größerem Maße bei Versuchen, bei denen Metopiron über ein bis zwei Tage eingenommen wird, so da $\beta$ der hier beschriebene Metopironkurztest dem Langzeittest vorzuziehen ist. 


\section{Literatur}

1. LiDDLE, G. W., H. L. Estep, J. W. WENDALI jr., W. C. Wrlliams jr. und A. W. Townes, J. Clin. Endocr., Springfield 19, 875 (1959). - 2. BethGE, H., Klin. Wschr. 48, 317 (1970). 3. Strotr, C. A., C. D. West, K. Nakagawa, T. Kondo und F. H. Týler, J. clin. Endocr., Springfield 29, 6 (1969). - 4. Plager, J. E., K. G. Schmidt und W. J. Staubitz, J. clin. Endocr., Springfield 25, 499 (1965). - 5. Buunck, W. und J. R. BIERICH, Klin. Wschr. 46, 202 (1968). - 6. KAPLAN, N. M., J. clin. Endocr., Springfield 23, 945 (1963). - 5. SpRUNT, J. G., E. R. Rutherford und Don H. Nelson, Acta endocr., K'hvn 59, 447 (1968). - 8. Jubiz, W., R. A. Levinson, A. W. Merkie, C. D. West und F. H. TYLER, Endocrinology 86, 328 (1970). 9. Nugent, C. A. und D. M. MAYES, J. clin. Endocr., Springfield 26, 1116 (1966). - 10. Leclerce, R., G. Copinschi und J. R. M. FranCKson, Rev. Franç. Etudes Clin. Biol. XIV, 815 (1969). 11. Henke, W. J., R. P. Doe und M. E. Jakobson, J. clin. Endocr. Springfield 20, 1527 (1960). - 12. SrLber, R. und R. D. BusCH,
J. clin. Endocr.,. Springfield 16, 1333 (1956). - 13. Murphy, B. E. P., A. B. Hoon und C. J. Patree, Can. Med. Ass. J. 90, 775 (1964). - 14. Guillemant, S., P. Thomopoulos und P. Diesgrez, Ann. Biol. Clin., Paris 27, 65 (1969). - 15. HenNs, W., H. van BAelen und P. DE MOor, Clin. Chim. Acta, Amsterdam 18, 361 (1967). - 16. VermeUlen, A. und L. VerdoncK, persönl. Mitteil. 17. Bertins, J. Z., M. H. Shąw, A. Kowarskr und C. J. Mrgeon, Steroids 15, 765 (1970). - 18. Harris, J. J., C. HoEgrL und M. G. CRANE, J. clin. Endocr., Springfield '27, 106 (1967). - 19. STrotr, C. A., T. Yoshim und M. B. LIPSETr, J. clin. Invest. 47, 94a (1968), Abstract 280. - 20. MoncloA, F., F. G. Peron und R. J. Dorfman, Endocrinology 65, 717 (1959). - 21. LAMPEHintzen, D. A. V. M. und L. G. Hurs IN T'VELD, J. clin. Endoct. Springfield 15, 1152 (1955). - 22. MARKS, L. J. und J. H. Lefrin, J. clin. Endocr., Springfield 14, 1263 (1954). - 23. MURPHY, B. E. P., J. clin. Endocr., Springfield 27, 973 (1967).

Dr. H. K. Kley Prof. Dr. H. L. Krüskemper 3000 Hannover Roderbruchstr. 101 


\section{Meisterschüsse, die ins Schwarze treffeng}

...wie Meßergebnisse

die im Zentrum liegen.

Brand-Geräte verhelfen Ihnen dazu.

Sie sind Meisterleistungen

im Bau von Volumenmeßgeräten.

Darum ist die „Richtigkeit” gut.

Sehr gut.
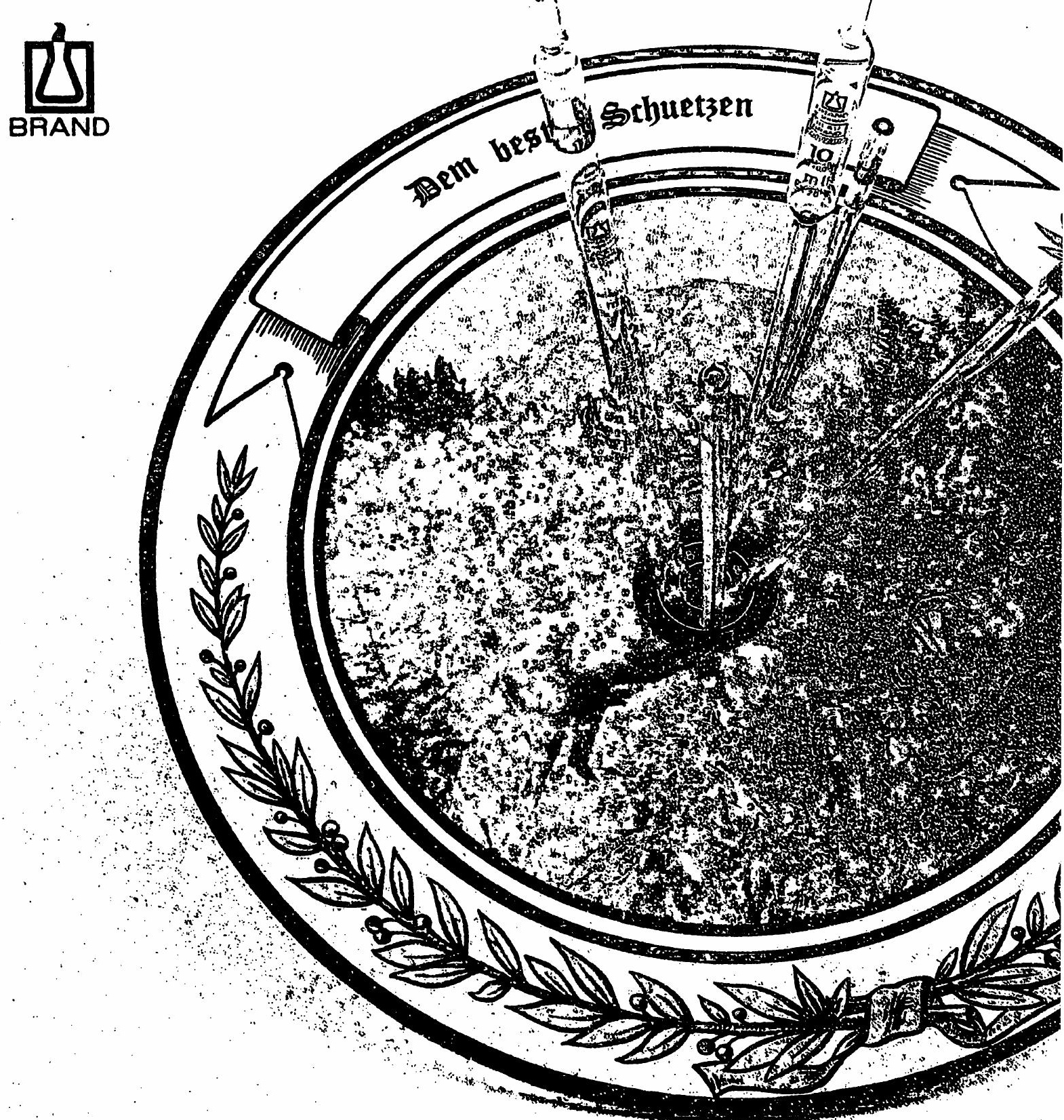

Mehr darüber 


\section{Richtigkeit von Messungen}

(Abweichung des Ist-Wertes vom Soll-Wert)

Die sog. "Richtigkeit” von Messungen gibt - neben der Aussage, daß Ergebnisse innerhalb von Fehlergrenzen liegen - die tatsächliche, durchschnittliche Abweichung des Ist-Wertes vom Soll-Wert an. Eine gute Richtigkeit ist Voraussetzung für hohe Reproduzierbarkeit von Meßergebnissen.

Bei Brand-Geräten wird diese Richtigkeit erreicht durch:

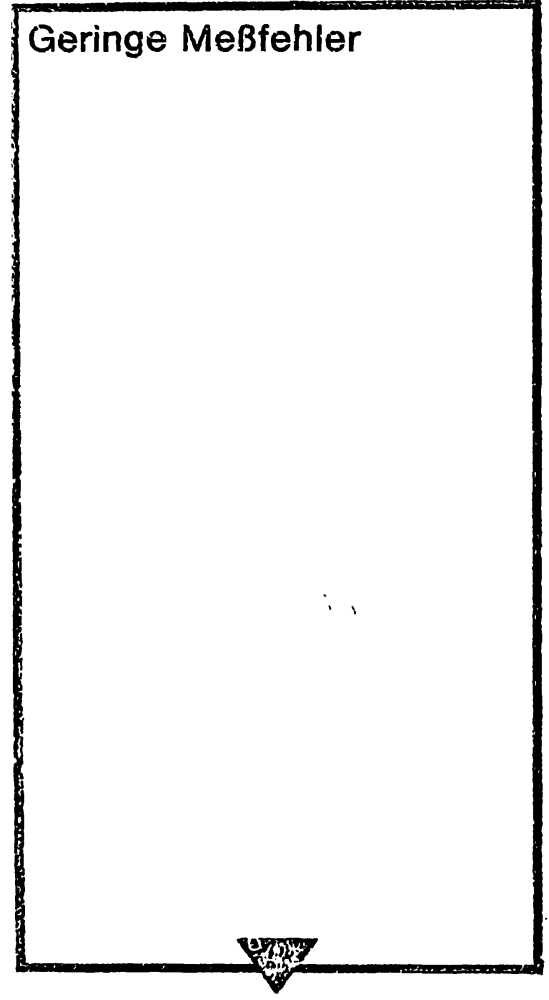

Leichte Ablesbarkeit
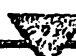

Lange Labensdauer

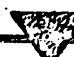

BRAND-GERÄTE

WEIL DIE

RENTABILITÄT

ENTSCHEIDET

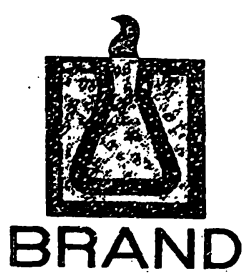

Gleichmäßige Querschnitte der in großen Serien automatisch heißverformten Ablauföffnungen (definierte Ablaufzeiten)

Bei Brand entwickelte Meßautomaten zur Festlegung der Volumenmarken

Zwischenwerte, die mit Hilfe dehnbarer Siebdruckschablonen genau abgeglichen sind

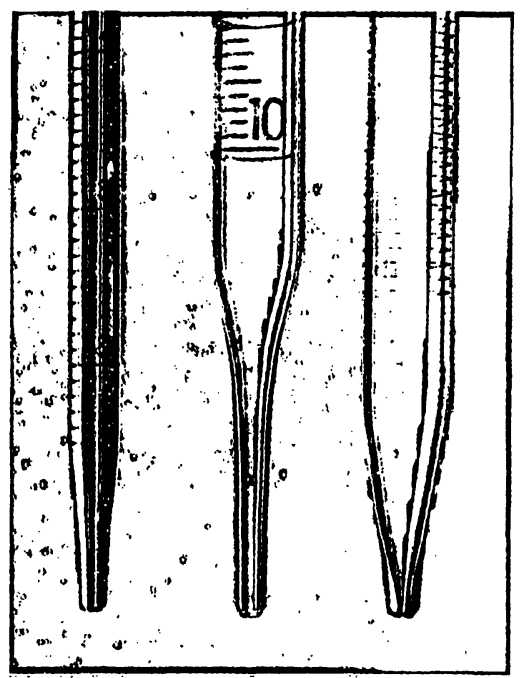

Scharfe Überwachung

jedes einzelnen

Fertigungsvorganges

Übersichtliche Skalen und Beschriftungen

Oberflächenstabilisierte Ablaufspitzen, robuste Bauweise und äußerst haltbare Graduierungen 